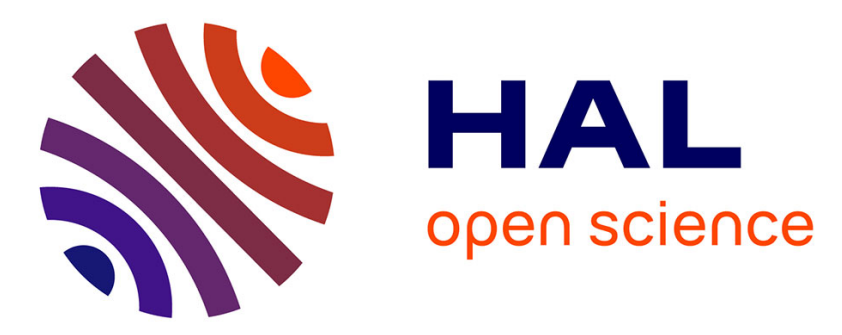

\title{
Aide à la décision en ingénierie inverse: une approche s'appuyant sur l'argumentation
}

\author{
Rallou Thomopoulos, Madalina Croitoru
}

\section{To cite this version:}

Rallou Thomopoulos, Madalina Croitoru. Aide à la décision en ingénierie inverse: une approche s'appuyant sur l'argumentation. Revue des Sciences et Technologies de l'Information - Série RIA : Revue d'Intelligence Artificielle, 2013, 27 (4-5), pp.493-513. 10.3166/ria.27.493-513 • lirmm-00835197

\section{HAL Id: lirmm-00835197 https://hal-lirmm.ccsd.cnrs.fr/lirmm-00835197}

Submitted on 7 Jan 2021

HAL is a multi-disciplinary open access archive for the deposit and dissemination of scientific research documents, whether they are published or not. The documents may come from teaching and research institutions in France or abroad, or from public or private research centers.
L'archive ouverte pluridisciplinaire HAL, est destinée au dépôt et à la diffusion de documents scientifiques de niveau recherche, publiés ou non, émanant des établissements d'enseignement et de recherche français ou étrangers, des laboratoires publics ou privés. 


\title{
Aide à la décision en ingénierie inverse
}

\section{Une approche s'appuyant sur l'argumentation}

\author{
Rallou Thomopoulos ${ }^{1,2}$, Madalina Croitoru ${ }^{2}$
}

1. INRA, UMR IATE

2 place Viala, 34060 Montpellier cedex 1, France

Rallou.Thomopoulos@supagro.inra.fr

\author{
2. INRIA GraphIK, LIRMM \\ 161 rue Ada, 34095 Montpellier cedex 5, France \\ Madalina.Croitoru@lirmm.fr
}

\begin{abstract}
RÉSUMÉ. L'évaluation de la qualité alimentaire est un processus complexe car il repose sur de nombreux critères historiquement regroupés en quatre grands types : qualité nutritionnelle, sensorielle, de praticité et d'hygiène. Ceux-ci peuvent être complétés par d'autres préoccupations émergentes telles que l'impact environnemental, les phénomènes économiques, etc. Toutefois, tous ces aspects de la qualité et leurs différentes composantes ne sont pas toujours compatibles et leur amélioration simultanée est un problème qui parfois n'a pas de solution évidente, ce qui correspond à un véritable verrou pour la prise de décision. Cet article propose une méthode d'aide à la décision en ingénierie inverse, i.e. guidée par les objectifs à l'aval de la filière. Elle se traduit par une approche en chaînage arrière s'appuyant sur l'argumentation.

ABSTRACT. Evaluating food quality is a complex process since it relies on numerous criteria historically grouped into four main types: nutritional, sensorial, practical and hygienic qualities. They may be completed by other emerging preoccupations such as the environmental impact, economic phenomena, etc. However, all these aspects of quality and their various components are not always compatible and their simultaneous improvement is a problem that sometimes has no obvious solution, which corresponds to a real issue for decision making. This paper proposes a decision support method in reverse engineering, i.e. guided by the objectives defined for the end products of an agrifood chain. It is materialized by a backward chaining approach based on argumentation.

MOTS-CLÉS : aide à la décision, représentation des connaissances, argumentation, ingénierie inverse, chaînage arrière, pilotage de filière agroalimentaire.

KEYWORDS: decision support, knowledge representation, argumentation, reverse engineering, backward chaining, agrifood chain control.
\end{abstract}

Revue d'intelligence artificielle $-\mathrm{n}^{\circ} 4-5 / 2013,493-513$ 


\section{Introduction}

L'offre au sein d'une filière agroalimentaire se construit traditionnellement des producteurs vers les consommateurs - en passant par les étapes intermédiaires de transformation, stockage, transport, conditionnement ... - c'est-à-dire de l'amont vers l'aval. Plus récemment, les contraintes de qualité s'accroissant, la notion de pilotage de filière par l'aval a émergé. C'est cette fois la demande - et non l'offre - qui fixe le cahier des charges des produits et c'est à la filière de s'adapter et de trouver les moyens d'y répondre. Les méthodes permettant, en partant de critères-cibles souhaités pour le produit final, d'identifier des moyens d'y parvenir en termes d'intervention sur les différentes étapes de la filière, sont qualifiées d' "ingénierie inverse ».

L'ingénierie inverse est connue au sein des filières pour poser de réels verrous méthodologiques à sa mise en place. Pour quelles raisons? Du point de vue des systèmes d'information, il n'existe pas réellement d'obstacle à retrouver l'information concernant les moyens de parvenir à une fin, si celle-ci est connue dans l'autre sens, le sens chronologique de la filière, des procédés vers le résultat. Les verrous sont en fait de deux types : le premier est la difficulté à définir le cahier des charges escompté pour le produit fini, les critères de qualité étant multiples, discutables et pas nécessairement compatibles entre eux. Comment dans ces conditions fixer des priorités pour les caractéristiques du produit fini? La seconde difficulté réside dans le fait que l'impact des différentes étapes d'une filière sur le produit final n'est pas complètement connu, même dans le sens de déroulement de la chaîne de production. Certaines étapes sont plus étudiées que d'autres, plusieurs étapes successives peuvent avoir des effets opposés dont on ne connaît pas l'effet résultant, les critères-cibles peuvent être en dehors des caractéristiques des produits issus des filières traditionnelles. Tous ces éléments constituent des freins à la mise en place de l'ingénierie inverse.

Dans cet article, nous abordons deux questions de cette problématique. Tout d'abord nous acceptons l'idée selon laquelle un cahier des charges unique ne peut pas être établi et que plusieurs points de vue complémentaires - éventuellement contradictoires - peuvent être exprimés (nutritionnel, environnemental, gustatif, etc.). Il s'agit ensuite d'évaluer leur compatibilité (ou incompatibilité) et d'identifier des solutions satisfaisant un ensemble maximal de points de vue.

Pour atteindre cet objectif, une bonne connaissance de l'organisation des filières agroalimentaires est nécessaire. Celles-ci ont évolué en quelques décennies en une industrie dynamique tournée vers l'innovation, afin de répondre à la demande en produits à la fois sûrs, sains et attractifs. Elles s'appuient sur les connaissances issues de leur savoir-faire mais aussi sur les résultats de la recherche. Celle-ci s'est construite essentiellement sur une approche analytique, fondée sur l'expérimentation et généralisée par des modèles mathématiques. Ces modèles, lorsqu'ils sont disponibles, peuvent alors être utilisés en simulation. Toutefois ils ne traitent pas la question de l'ingénierie inverse qui permet de partir de la demande. De plus l'arbitrage à l'échelle de la filière, contrairement à cette approche, est de nature qualitative, sous peine de s'avérer trop partielle et spécifique. Nous faisons donc ici l'hypothèse qu'une démarche qualitative 
en ingénierie inverse est possible. Nous proposons un cadre logique s'appuyant sur l'argumentation et introduisons une méthode de décision mettant en œuvre le châ̂nage arrière. L'approche, totalement générique, est illustrée dans le cas de la filière boulangère, suite au débat autour du changement de la teneur en cendres de la farine utilisée pour le pain de consommation courante, ainsi que de la teneur en sel, dans le cadre des recommandations du PNNS (Programme national nutrition-santé) émanant du ministère de la Santé.

L'article est organisé de la façon suivante. La section 2 introduit les éléments du formalisme logique. La section 3 présente la modélisation du problème. La section 4 expose la démarche d'aide à la décision. Les sections 5 et 6 proposent une discussion et concluent.

\section{Les éléments du formalisme}

\subsection{Pourquoi un langage logique?}

Nous attendons du langage de représentation utilisé qu'il permette d'effectuer des raisonnements : à partir des connaissances initiales, explicitement représentées, produire des connaissances et en particulier ici faire de la déduction, répondre à une requête, calculer des contradictions. Le choix d'un langage logique permet à de tels raisonnements d'être bien-fondés.

Par rapport à la logique des propositions, la logique du premier ordre permet de structurer les énoncés utilisés en exprimant des relations entre entités : elle fait apparaître les notions de prédicat, de constante, de variable et introduit des quantificateurs ("pour tout", "il existe"). Cette structuration permet d'affiner la représentation des connaissances et les raisonnements de façon utile à l'application : on peut ainsi représenter un pain comme une entité, exprimer des propriétés valables pour tous les pains, etc. Des exemples seront donnés tout au long des sections suivantes.

Dans la suite de cette section, nous introduisons les principales notions de notre langage logique suivant les notations de (Chein, Mugnier, 2009). Notons que nous n'utilisons pas de règles introduisant des variables existentielles en conclusion, pour deux raisons. D'une part, parce que ce type de règles ne s'est jamais présenté dans le cas d'étude considéré. D'autre part, parce que de telles règles compliqueraient les dérivations inverses utilisées dans ce papier et présentées dans la section suivante.

\subsection{Définitions en logique du premier ordre}

Soit $\mathbf{C}$ un ensemble de constantes et $\mathbf{P}=P_{1} \cup P_{2} \ldots \cup P_{n}$, où les $P_{i}$ sont des ensembles de prédicats d'arité commune $i$. Soit $\mathbf{V}$ un ensemble infini dénombrable de variables. Nous définissons l'ensemble des termes par $\mathbf{T}=\mathbf{V} \cup \mathbf{C}$. Suivant la dénomination usuelle, étant donnés $i \in\{1 \ldots n\}, p \in P_{i}$ et $t_{1}, \ldots, t_{i} \in \mathbf{T}$ nous appelons $p\left(t_{1}, \ldots, t_{i}\right)$ un atome. Soit $\gamma$ une conjonction d'atomes. Nous notons $\operatorname{var}(\gamma)$ l'ensemble des variables de $\gamma$. Etant donnés $\mathbf{V}, \mathbf{C}$ et $\mathbf{P}$, un fait sur $\mathbf{V}$ est la fermeture 
existentielle d'une conjonction d'atomes sur V (une $\exists \wedge$ formule). Etant donné un ensemble de faits $S$, nous notons $\bigwedge S$ la fermeture existentielle de la conjonction de tous les faits de $S$. Soulignons qu'il n'y a ni négation ni disjonction dans les faits.

Une interprétation de $(\mathbf{P}, \mathbf{C})$ est un couple $I=\left(\triangle, .^{I}\right)$ où $\triangle$ est le domaine d'interprétation (éventuellement infini) et ${ }^{I}$, la fonction d'interprétation, satisfait (a) $\forall c \in \mathbf{C}, c^{I} \in \triangle$, (b) $\forall i, \forall p \in P_{i}, p^{I} \subseteq \triangle^{i}$; et (c) $\forall\left(c, c^{\prime}\right)$ constantes distinctes de $\mathbf{C}$, $c^{I} \neq c^{I}$.

Exemple 1. - Pain, Céréale, PeuSalé, SansContaminants sont des exemples de prédicats unaires (d'arité 1) et EstIngrédient un exemple de prédicat binaire (d'arité 2) dans l'application.

blé, avoine, seigle, orge sont des exemples de constantes.

Céréale(blé) est un atome.

$\exists x(\operatorname{Pain}(x) \wedge$ EstIngrédient(blé, $x))$ est un exemple de fait.

\subsection{Conséquence logique, substitution et homomorphisme}

Nous notons $\models$ la conséquence logique classique de la logique du premier ordre.

Etant donnés un ensemble de variables $\mathbf{X}$ et un ensemble de termes $\mathbf{T}$, une substitution $\sigma$ de $\mathbf{X}$ par $\mathbf{T}$ est une application de $\mathbf{X}$ dans $\mathbf{T}$ (notée $\sigma: \mathbf{X} \rightarrow \mathbf{T}$ ). Etant donnée une conjonction d'atomes $\gamma, \sigma(\gamma)$ désigne la conjonction d'atomes obtenue à partir de $\gamma$ en remplaçant chaque occurrence de $x \in \mathbf{X} \cap \operatorname{var}(\gamma) \operatorname{par} \sigma(x)$. Si un fait $F$ est la fermeture existentielle d'une conjonction d'atomes $\gamma$ alors $\sigma(F)$ est la fermeture existentielle de $\sigma(\gamma)$. Enfin, un homomorphisme d'un fait $F$ vers un fait $F^{\prime}$ est une substitution $\sigma$ de $\operatorname{var}(F)$ par un sous-ensemble des termes de $F^{\prime}$ tel que $\sigma(F) \subseteq F^{\prime}$. L'homomorphisme peut aussi être défini sur les hypergraphes correspondant aux faits. Le théorème fondamental (Chein, Mugnier, 2009) établit que $F^{\prime} \models F$ si et seulement si il existe un homomorphisme de $F$ dans $F^{\prime}$.

EXEMPLE 2. - $($ Pain(banette $) \wedge$ EstIngrédient(blé, banette $))$ est une substitution de $(\operatorname{Pain}(x) \wedge$ EstIngrédient(blé, $x))$, où banette est une constante.

\subsection{Règles et dérivation}

Définissons maintenant les règles qui permettront d'enrichir les faits ci-dessus par de nouveaux faits (si elles sont applicables). Une règle $R$ est une formule $\forall X \forall Y$ $(H[X, Y] \rightarrow C[Y])$ où $H$, l'hypothèse, et $C$, la conclusion, sont deux conjonctions d'atomes; $X$ et $Y$ sont les ensembles de variables apparaissant respectivement dans $H$ et/ou $C$.

EXEMPLE 3. - Un exemple de règle est le suivant :

$\forall x(\operatorname{Pain}(x) \wedge$ SansPesticides $(x) \wedge$ SansMycotoxines $(x) \rightarrow$ SansContaminants $(x))$. 
Une règle $R=(H, C)$ est applicable à un fait $F$ s'il existe un homomorphisme $\pi$ de $H$ dans $F$. Dans ce cas, l'application de $R$ dans $F$ conformément à $\pi$ produit un fait $F \cup \pi(C)$. On dit alors que le nouveau fait (qui inclut l'ancien et y ajoute de l'information nouvelle) est une dérivation immédiate de $F$ par $R$, également notée abusivement $R(F)$.

Notons que cette technique est couramment utilisée, par exemple, pour la réponse à une requête en chaînage arrière (Van Melle et al., 1981; Clocksin, Mellish, 1984; Baget, Salvat, 2006), où une requête est réécrite conformément aux règles (puis une occurrence de la requête réécrite est recherchée dans les faits de la base de connaissances). Le même mécanisme est également abordé par les algorithmes de raisonnement abductif (Klarman et al., 2011) où un ensemble de faits minimal (au sens de l'inclusion) doit être ajouté à la base de connaissances pour pouvoir déduire la requête.

Dans ce papier nous ne nous intéressons pas nécessairement à la minimalité de ces ensembles, du fait de la petite taille des bases de connaissances employées. De plus, la sémantique obtenue par les extensions du système d'argumentation est similaire. Ce point pourrait toutefois constituer un fil conducteur intéressant pour les travaux à venir, afin d'optimiser notre méthode à des cas d'utilisation plus larges.

EXEMPLE 4. - Soit :

$F=$ Pain(bleuette) $\wedge$ SansPesticides(bleuette) $\wedge$ SansMycotoxines(bleuette)

et $R$ la règle de l'Exemple 3 .

$R$ est applicable à $F$ et produit par dérivation le nouveau fait suivant : Pain (bleuette) $\wedge$ SansPesticides (bleuette) $\wedge$ SansMycotoxines $($ bleuette $) \wedge$ SansContaminants(bleuette).

De façon similaire, une règle $R=(H, C)$ est inversement applicable à un fait $F$ s'il existe un homomorphisme $\pi$ de $C$ dans $F$. Dans ce cas, l'application inverse de $R$ dans $F$ conformément à $\pi$ produit un nouveau fait $F^{\prime}$ tel que $R\left(F^{\prime}\right)=F$. On dit alors que le nouveau fait est dérivation inverse immédiate de $F$ par $R$, également notée abusivement $R^{-1}(F)$.

EXEMPLE 5. - Soit :

$F=$ Pain(bleuette) $\wedge$ SansContaminants(bleuette)

et $R$ la règle de l'Exemple 3 .

$R$ est inversement applicable à $F$ et produit par dérivation inverse :

$F^{\prime}=$ Pain(bleuette) $\wedge$ SansPesticides(bleuette) $\wedge$ SansMycotoxines(bleuette).

Soit $F$ un fait et $\mathcal{R}$ un ensemble de règles. Un fait $F^{\prime}$ est appelé $\mathcal{R}$-dérivation de $F$ s'il existe une séquence finie (appelée séquence de dérivation) $F=F_{0}, F_{1}, \ldots, F_{k}=$ $F^{\prime}$ telle que pour tout $1 \leq i \leq k$ il existe une règle $R=(H, C) \in \mathcal{R}$ telle que $F_{i}$ est une dérivation immédiate de $F_{i-1}$ par $R$. L'ensemble de tous les faits obtenus à partir d'un fait $F$ et d'un ensemble de règles $\mathcal{R}$ par toutes les $\mathcal{R}$-dérivations est noté $\mathcal{R}(F)$. De même, partant d'un ensemble de faits $\mathcal{F}$, l'ensemble de tous les faits obtenus à 
partir de $\mathcal{F}$ et de $\mathcal{R}$ par toutes les $\mathcal{R}$-dérivations est noté $\mathcal{R}(\mathcal{F})$. Soulignons que, étant donnée la forme des règles, les dérivations ne sont pas infinies.

De façon similaire, nous pouvons définir des $\mathcal{R}$-dérivations inverses de $F$ et, étant donné $F$ et un ensemble de règles $\mathcal{R}$, toutes les $\mathcal{R}$-dérivations inverses de $F$ (noté $\left.\mathcal{R}^{-1}(F)\right)$.

Etant donnée la forme des règles dans le cas d'étude, ainsi que le nombre limité de faits et de règles, nous n'optimisons pas ce processus par une étude de minimalité (comme (Klarman et al., 2011)). Nous projetons d'approfondir ces aspects par la suite comme discuté dans la dernière section du papier.

\subsection{Expression de l'inconsistance}

Nous considérons une forme particulière de négation exprimée au moyen de contraintes négatives : elle permet d'exprimer une information qui ne doit pas pouvoir se déduire de la base de connaissances. L'objectif des contraintes négatives est d'exprimer des inconsistances dans les faits (y compris des faits partiellement générés par des règles). Plus précisément, une contrainte est une formule $\neg N$ où $N$ est un fait ( $\exists \wedge$ formule). Un ensemble de contraintes négatives noté $\mathcal{N}$ est un ensemble de négations de faits ( $\exists \wedge$ formules). Notons qu'une contrainte négative peut aussi être vue comme une règle de la forme $(N, \perp)$. Une contrainte négative est satisfaite par un fait $F$ si $F \not \models N$. Si un fait satisfait une contrainte négative alors il est consistant. Sinon le fait est inconsistant.

EXEMPle 6. $-N=\exists x(\operatorname{Augmentation}(x) \wedge \operatorname{Diminution}(x))$ est un exemple de contrainte négative.

Une contrainte négative est satisfaite par un fait $F$ et un ensemble de règles $\mathcal{R}$ si $\mathcal{R}(F) \not=N$. Dans ce cas on dit que le fait est $\mathcal{R}$-consistant (sinon il est $\mathcal{R}$ inconsistant). De même un ensemble de faits $\mathcal{F}$ est dit $\mathcal{R}$-consistant $\operatorname{si} \mathcal{R}(\mathcal{F}) \forall N$ (sinon il est $\mathcal{R}$-inconsistant).

\subsection{Base de connaissances consistante}

Une base de connaissances sur un vocabulaire est un triplet $\mathcal{K}=(\mathcal{F}, \mathcal{R}, \mathcal{N})$ composé de trois ensembles finis de formules :

- un ensemble $\mathcal{F}$ de faits, représentant des connaissances assertionnelles positives ;

- un ensemble $\mathcal{R}$ de règles, représentant des connaissances ontologiques pouvant être appliquées aux faits pour obtenir de nouveaux faits ;

- un ensemble $\mathcal{N}$ de négations de $\exists \wedge$ formules, représentant des contraintes négatives que les faits doivent satisfaire avant ou après l'application des règles. 
Une telle base de connaissances est consistante si $(\mathcal{F}, \mathcal{R})$ satisfait chaque contrainte de $\mathcal{N}$ (tous les faits de $\mathcal{F}$ sont $\mathcal{R}$-consistants).

EXEMPLE 7. - Soit $\mathcal{K}=(\mathcal{F}, \mathcal{R}, \mathcal{N})$ avec :

- $\mathcal{F}$ constitué des faits suivants :

$F_{1}=$ Pain(bleuette) $\wedge$ SansContaminants(bleuette)

$F_{2}=\exists$ e TauxExtraction(e,bleuette)

$F_{3}=\exists f($ TeneurFibres $(f$,bleuette $) \wedge$ Elevé $(f))$

$-\mathcal{R}$ constitué des trois règles suivantes :

$R_{1}=\forall x, y(\operatorname{Pain}(x) \wedge \operatorname{TauxExtraction}(y, x) \wedge \operatorname{SansPesticides}(x) \rightarrow \operatorname{Diminution}(y))$

$R_{2}=\forall x, y, z(\operatorname{Pain}(x) \wedge$ TauxExtraction $(y, x) \wedge$ TeneurFibres $(z, x) \wedge$ Elevé $(z) \rightarrow$ Augmentation $(y))$

$R_{3}=\forall x(\operatorname{Pain}(x) \wedge$ SansContaminants $(x) \rightarrow \operatorname{SansPesticides}(x) \wedge$ SansMycotoxines $(x))$

$-\mathcal{N}$ contenant la contrainte négative suivante : $N=\exists x$ (Augmentation $(x) \wedge \operatorname{Diminution}(x))$

$\mathcal{K}$ est inconsistant car $(\mathcal{F}, \mathcal{R}) \models N$. En effet $F_{1}$ et $R_{3}$ permettent de déduire SansPesticides(bleuette). Combiné à $F_{2}$ et $R_{1}$ on obtient Diminution(e). Or $F_{3}$ et $R_{2}$ conduisent à Augmentation(e), ce qui viole la contrainte négative $N$.

\subsection{Réponse à une requête : chaînage avant et chaînage arrière}

Un fait $Q$ est dérivé de $\mathcal{K}=(\mathcal{F}, \mathcal{R}, \mathcal{N})$ si et seulement si, soit $\mathcal{K}$ est inconsistant (et tout peut alors en être déduit), soit $(F, \mathcal{R}) \models Q$. Si $Q$ représente une requête, ce dernier cas signifie qu'il existe une réponse à la requête $Q$ dans $\mathcal{K}$.

Répondre à une requête $Q$ (représentée comme un fait dans le formalisme) relève de deux approches algorithmiques différentes : le chaînage avant ou le chaînage arrière. Les deux approches consistent respectivement à :

1. trouver une réponse à $Q$ dans les $\mathcal{R}$-dérivations des faits de la base de connaissances ;

2. calculer les $\mathcal{R}$-dérivations inverses de la requête et déterminer s'il existe une substitution dans les faits.

C'est cette seconde approche qui va nous intéresser par la suite.

\section{Modéliser le problème}

\subsection{Présentation du cas d'étude}

Le cas d'étude concerne initialement le débat autour du changement de la teneur en cendres de la farine utilisée pour le pain de consommation courante (pain français). Différents acteurs de la filière sont concernés, notamment le ministère de la 
Santé au travers de ses recommandations dans le cadre du PNNS (Programme national nutrition-santé), les meuniers, les boulangers, les nutritionnistes et les consommateurs.

En effet, le PNNS recommande de privilégier les produits céréaliers complets et en particulier de passer à un pain de consommation courante de type T80, i.e. fabriqué à partir de farine contenant un taux de cendres (taux de matière minérale) de $0.8 \%$, au lieu du type T65 (0.65\% de matière minérale) actuellement utilisé. Augmenter le taux de cendres revient effectivement à utiliser une farine plus complète, la matière minérale étant concentrée dans les couches périphériques du grain de blé, de même que bon nombre de constituants d'intérêt nutritionnel (vitamines, fibres, ...). Toutefois, les couches périphériques du grain sont aussi les plus exposées aux produits phytosanitaires, ce qui ne les rend pas recommandables d'un point de vue sanitaire, à moins d'utiliser de la farine biologique.

D'autres arguments, de différentes natures, sont en faveur ou défaveur d'un pain plus complet. D'un point de vue organoleptique par exemple, celui-ci perd en « croustillant ». D'un point de vue nutritionnel, l'argument selon lequel les fibres sont bénéfiques pour la santé est discuté, certaines d'entre elles étant irritantes pour l'appareil digestif. D'un point de vue économique, les boulangers craignent une perte de bénéfices en vendant moins, car un pain plus complet augmente la satiété - ce qui est bénéfique d'un point de vue nutritionnel, pour la régulation de l'appétit et pour la lutte contre les déséquilibres et pathologies alimentaires. Cependant un pain plus complet nécessite également moins de farine et plus d'eau pour sa confection, ce qui en diminue le coût. Les meuniers craignent pour leur part une perte de technicité dans la confection des farines.

Au-delà de la polémique sur le choix entre deux alternatives (T65 ou T80), il s'agit ici de considérer le problème dans son ensemble. On peut ainsi distinguer les différents points de vue en jeu, identifier les caractéristiques-cibles souhaitables, estimer les moyens d'y parvenir - sans se restreindre à la problématique du type de farine - et déterminer les différentes options qui se dessinent.

\subsection{Exprimer les caractéristiques-cibles suivant différents points de vue}

Exprimer les caractéristiques-cibles - ou buts - suivant différents points de vue consiste, d'une part, à identifier les différentes facettes intervenant dans la construction de la qualité du produit : les points de vue, grandes catégories de critères en jeu telles que nutrition, qualité hédonique, environnement, technologie, etc. ; d'autre part, à les décliner selon leurs différentes composantes (critères tels que le taux ou la qualité des fibres, minéraux, vitamines, etc.) et définir les orientations souhaitables, c'està-dire les valeurs souhaitées pour ces critères. Ces valeurs sont exprimées de façon qualitative, indépendamment les unes des autres dans un premier temps.

Ces premières étapes ont été réalisées, dans notre étude, à partir de sources d'information complémentaires comprenant, des plus formelles aux moins formelles :

1. des articles scientifiques évalués par des pairs; 
2. des rapports techniques ou des informations publiées sur les sites web;

3. des conférences et réunions scientifiques autour de projets de recherche;

4. des connaissances expertes obtenues au moyen d'entretiens.

Les articles scientifiques que nous avons analysés incluent (Bourre et al., 2008; Slavin, Green, 2007; Dubuisson-Quellier, 2006; Ginon et al., 2009; Layat, 2011). (Bourre et al., 2008) compare les différents types de farines d'un point de vue nutritionnel. (Slavin, Green, 2007) étudie le lien entre les fibres et la satiété. (DubuissonQuellier, 2006; Ginon et al., 2009) traitent des comportements de consommation et du consentement à payer des consommateurs, en particulier concernant la baguette française lorsque l'information concernant le niveau de fibres est fournie, sur la base expérimentale et statistique d'études de panels de consommateurs. (Layat, 2011) fournit une synthèse sur les aspects nutritionnels de la consommation de pain et fait le lien avec les aspects technologiques.

Nous avons également examiné des rapports techniques disponibles sur les sites web officiels concernant la politique de santé publique (PNNS (documents statutaires), 2010 ; PNNS (site web), 2010), le projet européen Healthgrain sur l'amélioration de la nutrition et de la santé à travers les grains de céréales (Dean et al., 2007 ; HEALTHGRAIN, 2009), assisté à des projets et colloques français au sujet des mesures sanitaires, nutritionnelles, technologiques et organoleptiques des pains (DINABIO, 2008; CADINNO, 2008; AQUANUP, 2009 ; FCN, 2009).

Enfin, plusieurs entretiens ont été menés pour recueillir les connaissances expertes du domaine, et en particulier celles des spécialistes technologues de notre laboratoire.

Un extrait des résultats obtenus dans la filière boulangère est synthétisé par les figures 1 , pour les aspects nutritionnels, et 2 , pour les aspects organoleptiques. Tous les points de vue ne sont pas abordés ici par souci de simplicité.

\subsection{Formalisation des buts}

Soit $\mathcal{K}=(\mathcal{F}, \mathcal{R}, \mathcal{N})$ une base de connaissances consistante. Il s'agit en l'occurrence de la base de connaissances partagée et admise par l'ensemble des acteurs impliqués dans la construction de la qualité des produits de la filière (meuniers, boulangers, consommateurs, nutritionnistes, technologues, chercheurs, pouvoirs publics). Nous supposons que les règles et les contraintes négatives sont partagées. Cette hypothèse sera relâchée lors de travaux futurs.

Les buts des différents acteurs peuvent être vus comme un ensemble de conjonctions d'atomes fermées existentiellement. Nous les notons $G_{1}, G_{2}, \ldots, G_{n}$.

Soit $\mathcal{G}$ l'ensemble des buts $\mathcal{G}=\left\{G_{1}, G_{2}, \ldots, G_{n}\right\}$. Les buts correspondent - par une fonction $\kappa: \mathcal{G} \rightarrow 2^{\mathcal{V}}$ - à un ensemble de points de vue $\mathcal{V}$ (nutritionnel, organoleptique, etc.). Cette fonction peut assigner un but à un ou plusieurs points de vue et chaque point de vue peut être associé à un ou plusieurs buts. Etant donné un but 


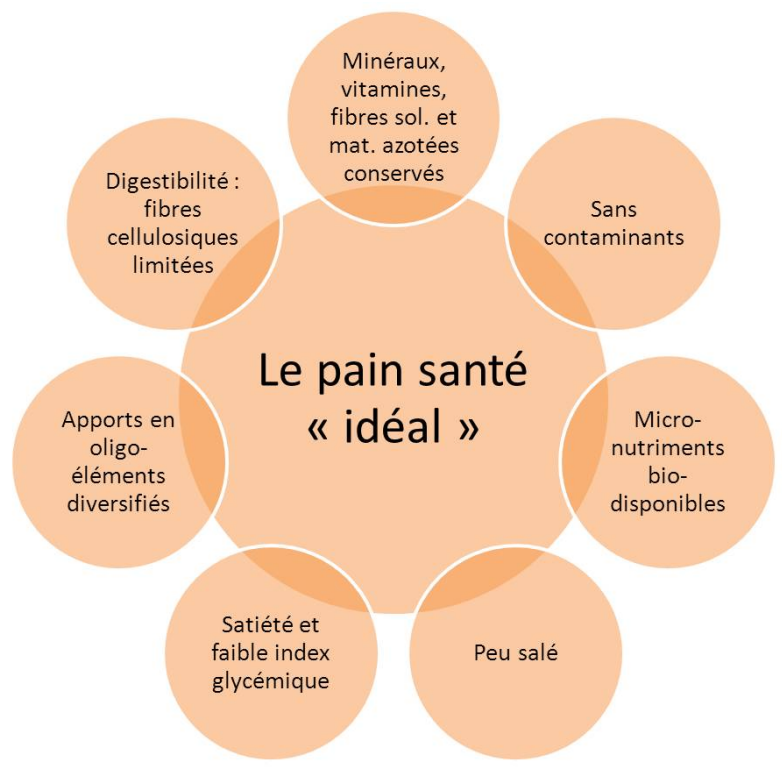

Figure 1. Buts nutritionnels

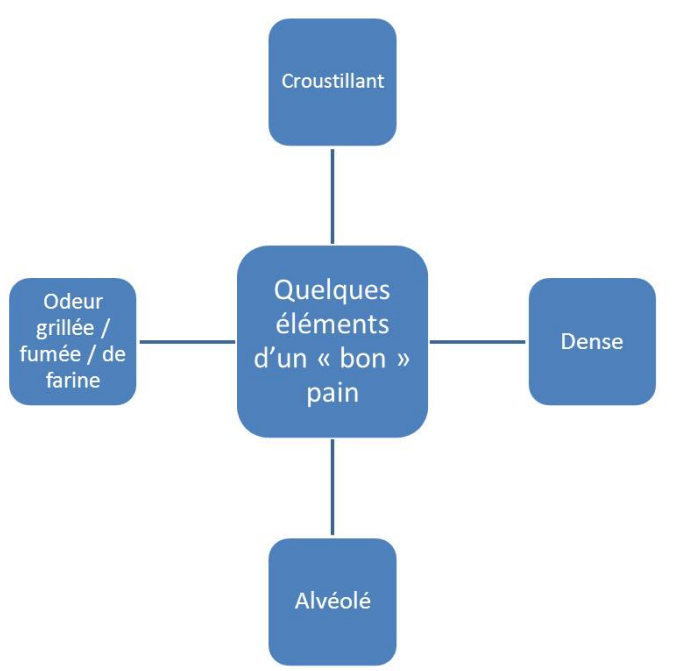

Figure 2. Buts organoleptiques 
$G_{i}$, l'ensemble des points de vue associés à ce but est noté $\kappa\left(G_{i}\right)$. De façon similaire, étant donné un point de vue $v_{i}$, l'ensemble des buts associés est noté $\kappa^{-1}\left(v_{i}\right)$.

EXEMPLE 8. - Soit l'ensemble de points de vue $\mathcal{V}=\{$ nutritionnel, sanitaire, organoleptique\} et $\mathcal{G}$ constitué des buts suivants :

$G_{1}=\exists x(\operatorname{Pain}(x) \wedge \operatorname{PeuSalé}(x))$

$G_{2}=\exists x(\operatorname{Pain}(x) \wedge$ SansContaminants $(x))$

$G_{3}=\exists x(\operatorname{Pain}(x) \wedge$ Croustillant $(x))$

$G_{4}=\exists x(\operatorname{Pain}(x) \wedge$ RicheOligoéléments $(x))$

On a $\kappa\left(G_{1}\right)=\kappa\left(G_{4}\right)=$ nutritionnel, $\kappa\left(G_{2}\right)=$ sanitaire et $\kappa\left(G_{3}\right)=$ organoleptique.

Inversement $\kappa^{-1}$ (nutritionnel $)=\left\{G_{1}, G_{4}\right\}, \kappa^{-1}$ (sanitaire) $=\left\{G_{2}\right\}$ et $\kappa^{-1}($ organoleptique $)=\left\{G_{3}\right\}$.

\subsection{Traduire l'ingénierie inverse}

L'ensemble de règles $\mathcal{R}$ de la base de connaissances $\mathcal{K}$ contient un ensemble de conditions suffisantes pour atteindre les buts $G_{i}$.

L'illustration sur la filière boulangère est schématisée par la figure 3 pour les aspects nutritionnels.

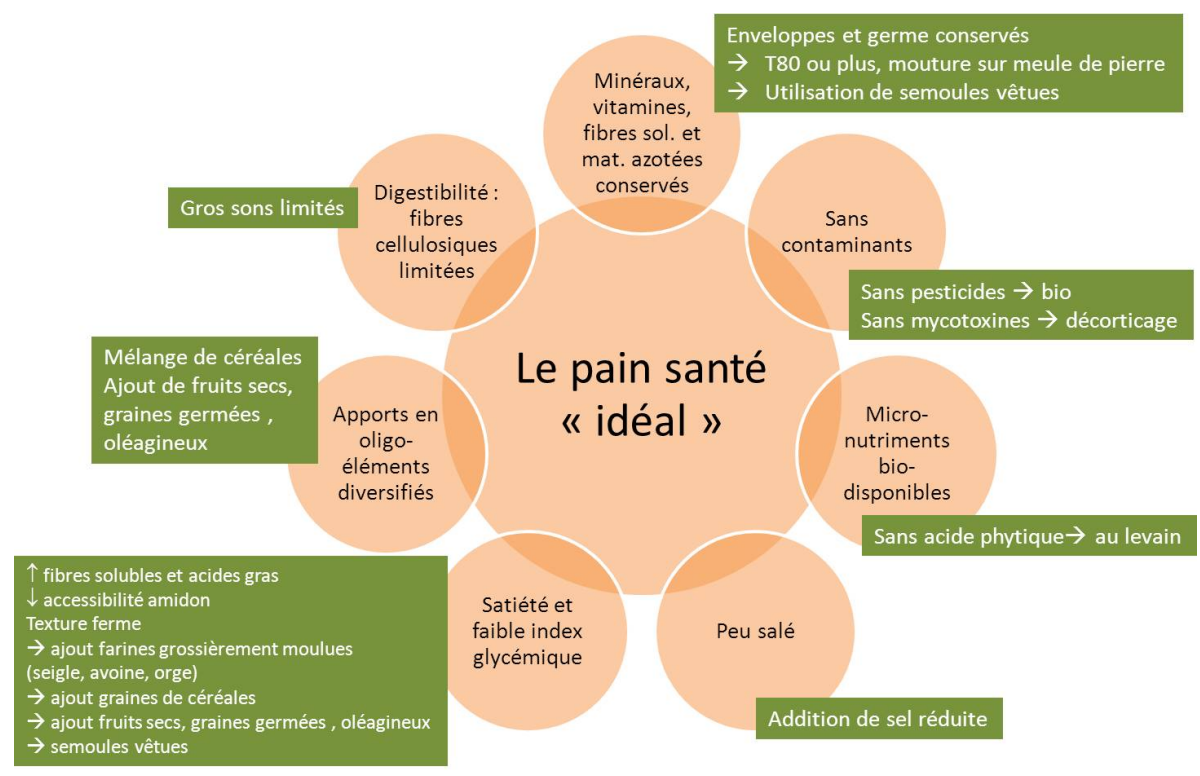

Figure 3. Moyens d'atteindre les buts nutritionnels 
EXEMPle 9. - Pour atteindre le but $G_{1}=\exists x(\operatorname{Pain}(x) \wedge \operatorname{PeuSalé(}(x))$, la base de connaissances $\mathcal{K}$ contient la règle suivante, qui exprime une condition suffisante :

$\forall x, y(\operatorname{Pain}(x) \wedge \operatorname{AdditionSel}(y, x) \wedge \operatorname{Diminution}(y) \rightarrow \operatorname{PeuSalé}(x))$

\subsection{Formalisation du processus d'ingénierie inverse}

Soit $G_{i}$ un but et $\mathcal{K}$ la base de connaissances. $\mathcal{K}$ est consistant et $G_{i}$ ne peut pas être dérivé de $\mathcal{K}$. Nous calculons les $\mathcal{R}$-dérivations inverses de $G_{i}$ (où $\mathcal{R}$ est l'ensemble des règles de la base de connaissances). Nous ajoutons tous les $\mathcal{R}^{-1}\left(G_{i}\right)$ aux faits. Nous obtenons ainsi une nouvelle base de connaissances $\mathcal{K}_{i}$ qui diffère de $\mathcal{K}$ uniquement par son ensemble de faits (qui maintenant inclut aussi $\mathcal{R}^{-1}\left(G_{i}\right)$ ) : $\mathcal{K}=\left(\mathcal{F} \cup \mathcal{R}^{-1}\left(G_{i}\right), \mathcal{R}, \mathcal{N}\right)$. Nous imposons également que $\mathcal{K}_{i}$ soit consistant.

Dans la suite du papier nous simplifions la notation de $\mathcal{F} \cup \mathcal{R}^{-1}\left(G_{i}\right)$ par $\mathcal{F}_{i}$ (l'ensemble des faits obtenus concernant le but $G_{i}$ ).

Considérons l'ensemble des buts $\mathcal{G}=\left\{G_{1}, G_{2}, \ldots, G_{n}\right\}$ et la base de connaissances initiale $\mathcal{K}=(\mathcal{F}, \mathcal{R}, \mathcal{N})$. Comme décrit ci-dessus nous calculons les $n$ bases de connaissances correspondant à chaque but : $\mathcal{K}_{i}=\left(\mathcal{F} \cup \mathcal{R}^{-1}\left(G_{i}\right), \mathcal{R}, \mathcal{N}\right)$ pour chaque $i=1, \ldots, n$. Considérons maintenant l'union de toutes ces bases de connaissances :

$$
\mathcal{K}_{a g g}=\left(\mathcal{F} \bigcup_{i=1, \ldots, n} \mathcal{R}^{-1}\left(G_{i}\right), \mathcal{R}, \mathcal{N}\right)
$$

\subsection{Exemple illustratif}

Illustrons la démarche sur l'exemple suivant.

EXEMPLE 10. - Soit $\mathcal{K}=(\mathcal{F}, \mathcal{R}, \mathcal{N})$ avec :

$-\mathcal{F}=\left\{F_{1}\right\}=\{$ TauxExtractionCourant $($ T65) $\}$

- $\mathcal{R}$ constitué des règles suivantes :

$R_{1}=\forall x, y(\operatorname{Pain}(x) \wedge \operatorname{TauxExtraction}(y, x) \wedge \operatorname{Diminution}(y) \rightarrow \operatorname{Digeste}(x))$

$R_{2}=\forall x, z(\operatorname{Pain}(x) \wedge$ AdditionSel $(z, x) \wedge \operatorname{Diminution}(z) \rightarrow \operatorname{PeuSalé}(x))$

$R_{3}=\forall x, y(\operatorname{Pain}(x) \wedge \operatorname{TauxExtraction}(y, x) \wedge$ Augmentation $(y) \rightarrow$ RicheOligoélé ments $(x)$ )

$R_{4}=\forall x, y(\operatorname{Pain}(x) \wedge \operatorname{TauxExtraction}(y, x) \wedge \operatorname{Diminution}(y) \rightarrow \operatorname{SansPesticides}(x))$

$-\mathcal{N}$ contenant la contrainte négative suivante :

$N=\exists x(\operatorname{Augmentation}(x) \wedge \operatorname{Diminution}(x))$

Soit $\mathcal{G}$ constitué des buts suivants :

$G_{1}=\exists p(\operatorname{Pain}(p) \wedge \operatorname{Digeste}(p)) \quad$ avec $\kappa\left(G_{1}\right)=$ nutritionnel

$G_{2}=\exists p(\operatorname{Pain}(p) \wedge \operatorname{PeuSalé}(p)) \quad$ avec $\kappa\left(G_{2}\right)=$ nutritionnel

$G_{3}=\exists p(\operatorname{Pain}(p) \wedge$ RicheOligoéléments $(p)) \quad$ avec $\kappa\left(G_{3}\right)=$ nutritionnel

$G_{4}=\exists p(\operatorname{Pain}(p) \wedge$ SansPesticides $(p)) \quad$ avec $\kappa\left(G_{4}\right)=$ sanitaire. 
On a $\mathcal{K}_{1}=\left(\mathcal{F}_{1}, \mathcal{R}, \mathcal{N}\right)$ avec $\mathcal{F}_{1}=\mathcal{F} \cup \mathcal{R}^{-1}\left(G_{1}\right)$ constitué des deux faits suivants : $F_{1}=$ TauxExtractionCourant $($ T65)

$F_{2}=\operatorname{Pain}(p) \wedge \operatorname{TauxExtraction}(\tau, p) \wedge \operatorname{Diminution}(\tau)$

$\mathcal{K}_{2}=\left(\mathcal{F}_{2}, \mathcal{R}, \mathcal{N}\right)$ avec $\mathcal{F}_{2}=\mathcal{F} \cup \mathcal{R}^{-1}\left(G_{2}\right)$ constitué des deux faits suivants :

$F_{1}=$ TauxExtractionCourant $($ T65)

$F_{3}=\operatorname{Pain}(p) \wedge \operatorname{AdditionSel}(s, p) \wedge \operatorname{Diminution}(s)$

$\mathcal{K}_{3}=\left(\mathcal{F}_{3}, \mathcal{R}, \mathcal{N}\right)$ avec $\mathcal{F}_{3}=\mathcal{F} \cup \mathcal{R}^{-1}\left(G_{3}\right)$ constitué des deux faits suivants : $F_{1}=$ TauxExtractionCourant $($ T65)

$F_{4}=\operatorname{Pain}(p) \wedge \operatorname{TauxExtraction}(\tau, p) \wedge \operatorname{Augmentation}(\tau)$

$\mathcal{K}_{4}=\left(\mathcal{F}_{4}, \mathcal{R}, \mathcal{N}\right)$ avec $\mathcal{F}_{4}=\mathcal{F} \cup \mathcal{R}^{-1}\left(G_{4}\right)$ constitué des deux faits suivants : $F_{1}=$ TauxExtractionCourant $($ T65)

$F_{2}=\operatorname{Pain}(p) \wedge \operatorname{TauxExtraction}(\tau, p) \wedge \operatorname{Diminution}(\tau)$

On obtient $\mathcal{K}_{\text {agg }}=\left(\mathcal{F} \bigcup_{i=1, \ldots, n} \mathcal{R}^{-1}\left(G_{i}\right), \mathcal{R}, \mathcal{N}\right)$ avec $\mathcal{F} \bigcup_{i=1, \ldots, n} \mathcal{R}^{-1}\left(G_{i}\right)=$ $\left\{F_{1}, F_{2}, F_{3}, F_{4}\right\}$.

Comme le montre l'exemple, il peut arriver que $\mathcal{K}_{\text {agg }}$ soit inconsistant (ainsi $F_{2}, F_{4}$ violent la contrainte négative $N$ ). C'est le cas même pour des buts appartenant au même point de vue (ainsi $F_{2}$ est obtenu pour $G_{1}$ et $G_{4}$, associés respectivement aux points de vue nutritionnel et sanitaire, et $F_{4}$ est obtenu pour $G_{3}$, associé au point de vue nutritionnel).

Afin d'isoler des sous-ensembles maximaux consistants nous utilisons l'argumentation (Dung, 1995) qui, au moyen des extensions, permet de les calculer. De plus, les extensions nous permettront de voir quels points de vue sont associés à chaque sousensemble maximal cohérent (au moyen de la fonction $\kappa$ ). On pourra alors utiliser soit des procédures de vote simple pour déterminer le point de vue à suivre, soit d'autres modes de sélection à base de préférences. Ces points sont explicités dans la section 4 .

\section{Aide à la décision}

Nous venons de voir qu'un ensemble de buts a été explicité. Puis un ensemble de faits, identifiés comme des conditions suffisantes pour atteindre ces buts, a été calculé. Il nous faut à présent définir les éléments d'un système d'argumentation (Dung, 1995) dans ce contexte, et en particulier ce qu'est un argument et ce qu'est une attaque. Ils serviront de base dans le processus d'aide à la décision.

\subsection{Calcul des arguments et des extensions}

Nous partons de $\mathcal{K}_{\text {agg }}$, l'union $\mathcal{R}$-inconsistante des $n$ bases de connaissances respectant chacune le même ensemble de règles et de contraintes mais ayant des faits $\mathcal{R}$-consistants différents. 
Un argument $a$ sur $\mathcal{K}_{a g g}$ est une séquence $a=\left(\varphi_{0}, \varphi_{1}, \ldots, \varphi_{k}\right)$ où $\varphi_{0}$ est un sous-ensemble de faits de $\mathcal{K}_{\text {agg }}, \varphi_{1}=\bigwedge \varphi_{0}$, tous les $\varphi_{i}(i=1, \ldots, k)$ sont des faits $\mathcal{R}$-consistants et, pour tout $i=2, \ldots, k, \exists R \in \mathcal{R}$ une règle telle que $\varphi_{i+1}=R\left(\varphi_{i}\right)$, i.e. $\varphi_{i+1}$ est une dérivation immédiate de $\varphi_{i}$.

EXEMPLE 11. - Reprenons la base de connaissances $\mathcal{K}_{a g g}$ de l'exemple 10. Un exemple d'argument est :

$a=\left(\left\{F_{2}\right\}, F_{2}, R_{1}\left(F_{2}\right)\right)$

avec $R_{1}\left(F_{2}\right)=\operatorname{Pain}(p) \wedge \operatorname{TauxExtraction}(\tau, p) \wedge \operatorname{Diminution}(\tau) \wedge \operatorname{Digeste}(p)$.

Soit $a=\left(\varphi_{0}, \ldots, \varphi_{k}\right)$ un argument. Nous notons $\operatorname{Supp}(a)=\varphi_{0}$ le support de $a$ et $\operatorname{Conc}(a)=\varphi_{k}$ la conclusion de $a$. Etant donnée la base de connaissances $\mathcal{K}_{\text {agg }}$ nous notons $\mathcal{A}$ l'ensemble des arguments construits à partir des faits de $\mathcal{K}_{\text {agg }}$. Nous définissons alors la relation d'attaque Att comme un sous-ensemble de $\mathcal{A} \times \mathcal{A}$. Soient $a$ et $b$ deux arguments. On dit que l'argument $a$ attaque l'argument $b$, c'està-dire $(a, b) \in A t t$, si et seulement si $\exists \varphi \in S u p p(b)$ tel que l'ensemble des faits $\{\operatorname{Conc}(a), \varphi\}$ est $\mathcal{R}$-inconsistant.

EXEMPLE 12. - Soit $a$ l'argument de l'exemple 11 et $b$ l'argument suivant : $b=\left(\left\{F_{4}\right\}, F_{4}, R_{3}\left(F_{4}\right)\right)$ avec $R_{3}\left(F_{4}\right)=\operatorname{Pain}(p) \wedge \operatorname{TauxExtraction}(\tau, p) \wedge$ Augmentation $(\tau) \wedge$ RicheOligoéléments $(p)$.

L'argument $a$ attaque l'argument $b$ car $\{\operatorname{Conc}(a), \bigwedge \operatorname{Supp}(b)\}=\left\{R_{1}\left(F_{2}\right), F_{4}\right\}$ viole la contrainte négative $N$.

Dans ce cadre, un système d'argumentation est un couple $(\mathcal{A}, A t t)$ où $\mathcal{A}$ est un ensemble d'arguments sur $\mathcal{K}_{a g g}$ et $A t t$ est la relation d'attaque définie sur cet ensemble.

EXemple 13. - Poursuivons l'exemple précédent. Nous considérons par la suite le système d'argumentation $(\mathcal{A}, A t t)$ où $\mathcal{A}$ est constitué des arguments suivants :

$a=\left(\left\{F_{2}\right\}, F_{2}, R_{1}\left(F_{2}\right)\right)$ avec $R_{1}\left(F_{2}\right)=\operatorname{Pain}(p) \wedge \operatorname{TauxExtraction}(\tau, p) \wedge$

$\operatorname{Diminution}(\tau) \wedge \operatorname{Digeste}(p)$

$b=\left(\left\{F_{4}\right\}, F_{4}, R_{3}\left(F_{4}\right)\right)$ avec $R_{3}\left(F_{4}\right)=\operatorname{Pain}(p) \wedge \operatorname{TauxExtraction}(\tau, p) \wedge$

Augmentation $(\tau) \wedge$ RicheOligoéléments $(p)$

$c=\left(\left\{F_{2}\right\}, F_{2}, R_{4}\left(F_{2}\right)\right)$ avec $R_{4}\left(F_{2}\right)=\operatorname{Pain}(p) \wedge \operatorname{TauxExtraction}(\tau, p) \wedge$ $\operatorname{Diminution}(\tau) \wedge$ SansPesticides $(p)$

$d=\left(\left\{F_{3}\right\}, F_{3}, R_{2}\left(F_{3}\right)\right)$ avec $R_{2}\left(F_{3}\right)=\operatorname{Pain}(p) \wedge \operatorname{AdditionSel}(s, p) \wedge \operatorname{Diminution}(s)$

$\wedge$ PeuSalé(p)

et $A t t=\{(a, b),(b, a),(b, c),(c, b)\}$.

REMARQue. - Notons que dans l'exemple ci-dessus la relation d'attaque est symétrique, ce qui n'est pas nécessairement le cas. Considérons les faits suivants :

$F_{1}^{\prime}=$ Pain(bleuette $) \wedge$ SansContaminants(bleuette)

$F_{2}^{\prime}=\exists f($ TeneurFibres $(f$,bleuette $) \wedge$ Elevé $(f))$,

les règles suivantes : 
$R_{1}^{\prime}=\forall x($ SansContaminants $(x) \rightarrow$ SansPesticides $(x) \wedge$ SansMycotoxines $(x))$

$R_{2}^{\prime}=\forall x, y($ TeneurFibres $(y, x) \wedge$ Elevé $(y)) \rightarrow$ ContientContaminants $\left.(x)\right)$

et la contrainte négative suivante :

$N^{\prime}=\exists x($ ContientContaminants $(x) \wedge$ SansContaminants $(x))$.

Soient $a^{\prime}$ et $b^{\prime}$ les arguments construits comme suit :

$a^{\prime}=\left(\left\{F_{1}^{\prime}\right\}, F_{1}^{\prime}, R_{1}^{\prime}\left(F_{1}^{\prime}\right)\right)$

$b^{\prime}=\left(\left\{F_{2}^{\prime}\right\}, F_{2}^{\prime}, R_{2}^{\prime}\left(F_{2}^{\prime}\right)\right)$ avec $R_{2}^{\prime}\left(F_{2}^{\prime}\right)=\exists f($ TeneurFibres $($ f,bleuette $) \wedge$ Elevé $(f) \wedge$

ContientContaminants(bleuette)).

L'argument $b^{\prime}$ attaque l'argument $a^{\prime}$ car $\left\{\operatorname{Conc}\left(b^{\prime}\right), \bigwedge S u p p\left(a^{\prime}\right)\right\}=\left\{R_{2}^{\prime}\left(F_{2}^{\prime}\right), F_{1}^{\prime}\right\}$ viole la contrainte négative $N^{\prime}$. On obtient une relation d'attaque non symétrique : $A t t^{\prime}=\left\{\left(b^{\prime}, a^{\prime}\right)\right\}$.

Rappelons enfin les principes sur lesquels s'appuie la notion de "cohérence" en argumentation et qui vont nous permettre, au moyen des extensions, de calculer les sous-ensembles d'arguments maximaux consistants dans la dernière étape de notre démarche.

Soit $(\mathcal{A}, A t t)$ un système d'argumentation, soient $\mathcal{B} \subseteq \mathcal{A}$ et $a \in \mathcal{A}$. On dit que $\mathcal{B}$ est sans conflit si et seulement si il n'existe pas d'arguments $a, b \in \mathcal{B}$ tels que $(a, b) \in A t t$. On dit que le sous-ensemble $\mathcal{B}$ défend l'argument $a$ si et seulement si pour tout argument $b \in \mathcal{A}$, si $(b, a) \in A t t$ alors il existe $c \in \mathcal{B}$ tel que $(c, b) \in \operatorname{Att}$. Le sous-ensemble $\mathcal{B}$ est dit admissible si et seulement si il est sans conflit et défend tous ses arguments.

Différentes sémantiques ont été définies pour la notion d'extension. $\mathcal{B}$ est une extension complète si et seulement si $\mathcal{B}$ défend tous ses arguments et contient tous les arguments qu'il défend. $\mathcal{B}$ est une extension préférée si et seulement si c'est un ensemble admissible maximal (au sens de l'inclusion ensembliste). $\mathcal{B}$ est une extension stable s'il est sans conflit et pour tout $a \in \mathcal{A} \backslash \mathcal{B}$, il existe un argument $b \in \mathcal{B}$ tel que $(b, a) \in A t t . \mathcal{B}$ est une extension semi-stable si et seulement si $\mathcal{B}$ est une extension complète et que l'union entre l'ensemble $\mathcal{B}$ et l'ensemble de tous les arguments attaqués par $\mathcal{B}$ est maximal (pour l'inclusion ensembliste). L'ensemble des extensions suivant une sémantique $x$ donnée est noté $\operatorname{Ext}_{x}(\mathcal{A}, A t t)$.

Comme antérieurement prouvé en logique propositionnelle (et facilement étendu au sous-ensemble de la logique du premier ordre étudié dans ce papier), les sémantiques stable, semi-stable et préférée correspondent aux sous-ensembles de connaissances consistants maximaux dans un système d'argumentation (Vesic, 2012). Parmi ces trois sémantiques, le choix de la sémantique préférée se justifie ici. En effet, les sémantiques stable et semi-stable sont fondées sur la capacité d'une extension à attaquer le maximum d'arguments extérieurs à cette extension. Or les attaques s'appuient dans ce papier sur la notion de contrainte négative. Choisir la sémantique stable ou semi-stable viserait à privilégier les extensions qui préconisent des actions ayant le plus d'incompatibilités avec celles des arguments extérieurs à l'extension, ce qui n'est pas recherché ici, au contraire. 
EXEMPLE 14. - Dans le système d'argumentation de l'exemple 13, les sémantiques stable, semi-stable et préférée se trouvent être confondues. On a :

$\operatorname{Ext}_{\text {stable }}(\mathcal{A}, A t t)=\operatorname{Ext}_{\text {semi-stable }}(\mathcal{A}, A t t)=\operatorname{Ext}_{\text {preferee }}(\mathcal{A}, A t t)=$ $\{\{a, c, d\},\{b, d\}\}$.

\subsection{Choix des points de vue à retenir}

Partant de l'ensemble des extensions $\operatorname{Ext}_{x}(\mathcal{A}, A t t)$, la démarche d'aide à la décision est la suivante :

1. Pour chaque extension $\varepsilon \in \operatorname{Ext}_{x}(\mathcal{A}, A t t)$ :

- considérer les faits apparaissant dans les arguments de $\varepsilon$;

- identifier les bases de connaissances $\mathcal{K}_{i}$ dont ces faits sont issus ;

- obtenir ainsi l'ensemble des buts $G_{i}$ satisfaits ;

- déduire l'ensemble des points de vue associés, à l'aide de la fonction $\kappa$;

- présenter aux experts l'ensemble des buts et des points de vue compatibles, correspondant à l'extension considérée.

2. On obtient de cette manière autant d'options qu'il y a d'extensions dans $\operatorname{Ext}_{x}(\mathcal{A}, A t t)$. Pour décider de l'option à retenir, plusieurs lignes de conduite sont alors envisageables et proposées aux experts. Le processus de décision peut notamment :

- suggérer l'option satisfaisant le plus grand nombre de buts ;

- suggérer l'option contribuant au plus grand nombre de points de vue ;

- demander à l'expert du domaine de définir une préférence sur les buts et/ou les points de vue considérés.

EXEMPLE 15. - Dans notre exemple la première extension $\{a, c, d\}$ s'appuie sur les faits $F_{2}$ et $F_{3}$, issus des bases de connaissances $\mathcal{K}_{1}, \mathcal{K}_{2}$ et $\mathcal{K}_{4}$ et satisfaisant les buts $G_{1}, G_{2}$ et $G_{4}$. Les buts $G_{1}$ et $G_{2}$ sont associés au point de vue nutritionnel, tandis que le but $G_{4}$ est associé au point de vue sanitaire.

La seconde extension $\{b, d\}$ s'appuie sur les faits $F_{3}$ et $F_{4}$, issus des bases de connaissances $\mathcal{K}_{2}$ et $\mathcal{K}_{3}$ et satisfaisant les buts $G_{2}$ et $G_{3}$, tous deux associés au point de vue nutritionnel.

La première option (correspondant à l'extension $\{a, c, d\}$ ) consisterait à réaliser les faits $F_{2}$ et $F_{3}$ et permettrait de satisfaire le plus grand nombre de buts et de prendre en compte le plus grand nombre de points de vue.

La seconde option (correspondant à l'extension $\{b, d\}$ ) consisterait à réaliser les faits $F_{3}$ et $F_{4}$. Elle satisfairait deux buts et prendrait en compte un seul point de vue. Elle peut toutefois être pertinente si le but $G_{3}$, qui n'est pas satisfait par la première option, est jugé prioritaire. 


\section{Synthèse et discussion}

\subsection{Schéma global de la démarche}

Une synthèse de la démarche proposée est schématisée sur la figure 4 :

- L'étape 0 désigne la formalisation des buts, des points de vue, et leur correspondance par la fonction $\kappa$ comme présenté dans la section 3.3.

- L'étape 1 correspond au calcul, par dérivation inverse, des bases de connaissances associées à chaque but (section 3.5). On considère alors leur union.

- L'étape 2 correspond au calcul des arguments et des extensions tel qu'exposé dans la section 4.1.

- Enfin l'étape 3 détermine le choix des points de vue conformément à la section 4.2 .

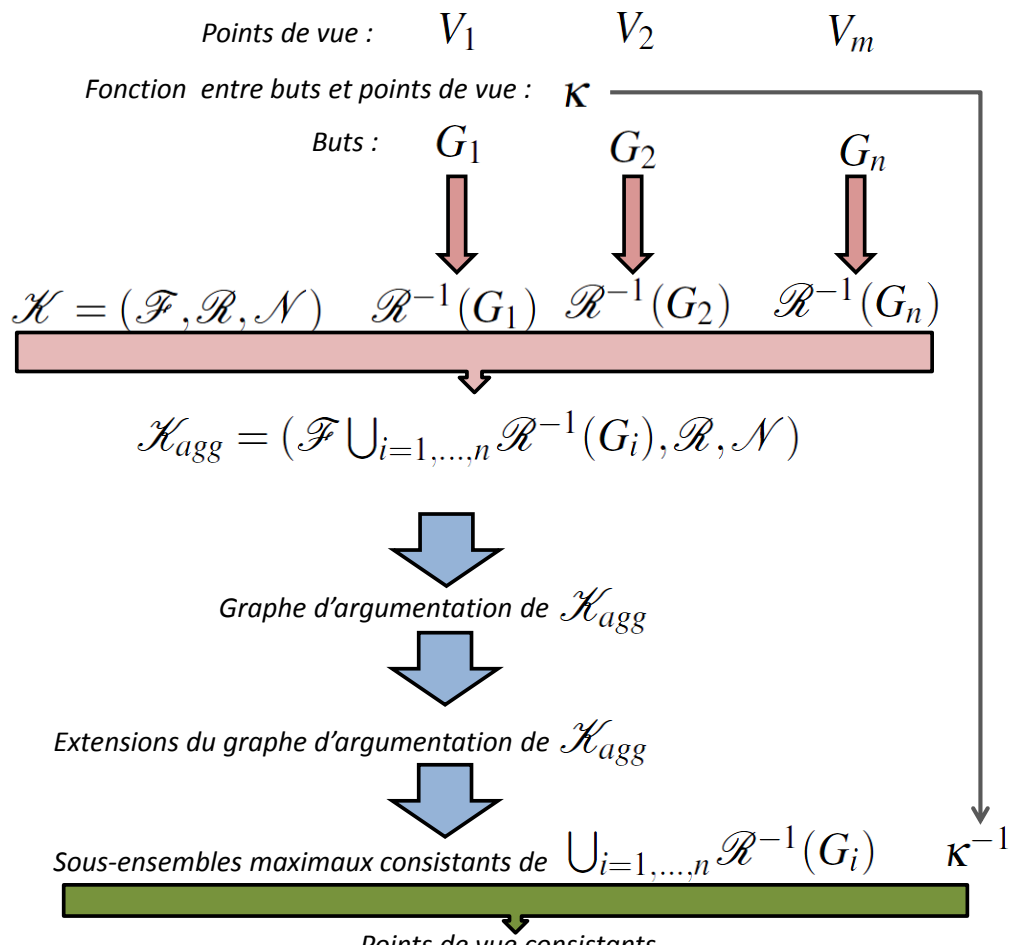

Etape 0

Etape 1

Etape 2

Points de vue consistants

Figure 4. Schéma global de la démarche

\subsection{Autres approches et positionnement}

La conciliation des différents points de vue en jeu au sein d'une filière soulève des questions encore peu abordées formellement. Les liens entre argumentation et déci- 
sion sont peu explorés dans la littérature, la recherche en décision argumentée ayant été amorcée assez récemment avec les travaux de (Amgoud, Prade, 2009). Il existe par ailleurs une communauté internationale active sur différents aspects de l'argumentation (Besnard, Hunter, 2008 ; Rahwan, Simari, 2009) d'une part, et sur la décision multicritère (Bouyssou et al., 2009) d'autre part. Cependant ces deux domaines ont essentiellement été abordés séparément jusqu'ici. Or la démarche paraît extrêmement pertinente pour aider à la prise de décision dans les filières agroalimentaires (Thomopoulos et al., 2009), dans un objectif d'arbitrage.

Le contexte d'application principal de l'argumentation - au sens large, et non au sens d'un cadre formel en informatique, initié par (Dung, 1995) - est historiquement le domaine judiciaire, bien que d'autres applications aient été abordées du point de vue du management ou de l'étude de débats en sciences de gestion (Maguire, Boiney, 1994 ; Gottsegen, 1998).

Récemment, les premiers travaux en argumentation pour la prise de décision appliqués à l'arbitrage au sein de la filière céréalière ont été réalisés (Bourguet, 2010 ; Bourguet et al., 2013). Ils s'appuient sur un modèle de décision argumentée pour proposer des recommandations de consommation destinées à des publics spécifiques (prévention cardiovasculaire, etc.). Le présent article aborde un verrou essentiel pour le pilotage de filière en y associant l'ingénierie inverse et introduit une démarche permettant de proposer des options en termes de procédés de fabrication. Notons qu'en revanche l'argumentation est utilisée ici comme mode de calcul - et non comme moyen d'analyse - des objectifs compatibles dans la filière.

Comme discuté dans les sections précédentes, la technique de chaînage arrière en elle-même est utilisée dans d'autres travaux tels que (Baget, Salvat, 2006; Klarman et al., 2011). L'approche présentée dans ce papier se rapproche de (Baget, Salvat, 2006), qui s'intéresse à la réponse à une requête, si l'on considère un but comme une requête et les moyens d'atteindre ce but comme une réponse. Cependant cette réponse n'est pas nécessairement dans la base de connaissances initiale mais calculée par dérivation inverse. La démarche présente aussi des points communs avec (Klarman et al., 2011), par le calcul d'un ensemble de faits minimal. Toutefois les ensembles de faits sont obtenus par les extensions, qui cherchent au contraire à maximiser l'ensemble des arguments compatibles.

Enfin une série de travaux tels que (Amgoud, Cayrol, 2002; Bench-Capon, 2003 ; Kaci, Torre, 2008 ; Amgoud et al., 2000 ; Bourguet et al., 2013) ont introduit dans les systèmes d'argumentation la notion de force des arguments, par le biais de préférences ou de valeurs associées aux arguments. En revanche ces travaux ne se situent pas dans une optique d'ingénierie inverse et ne manipulent pas les éléments du formalisme logique utilisé ici. Il serait de ce fait judicieux d'étudier l'introduction de préférences sur les buts, les règles ou les faits. L'expression de préférences pourrait alors participer à la résolution des conflits. 


\section{Conclusion et perspectives}

Cette étude de cas représente une application originale et une approche introspective dans le domaine agroalimentaire et l'aide à la décision pour les filières. Il nécessite néanmoins une tâche de modélisation des connaissances très coûteuse, qui ne peut en l'état être automatisée, et qui dépend fortement de la qualité de l'expertise et de l'élicitation (exhaustivité, certitude, etc.). La tendance des outils d'aide à la décision inclut de plus en plus les méthodes d'argumentation comme moyen d'impliquer les parties prenantes dans la tâche de modélisation et le processus décisionnel en favorisant leurs interactions.

Nous avons pris le parti dans cet article de nous focaliser sur la démarche adoptée, plutôt que de présenter l'application de façon exhaustive. Une phase visant à la fois à compléter les connaissances saisies et à évaluer l'intérêt du système est actuellement en cours, sous la forme d'une série d'ateliers permettant les échanges avec et entre les experts de la filière. Elle porte sur un nombre restreint de scénarios préalablement identifiés comme controversés. L'optimisation algorithmique est une perspective importante de ce travail. Un autre élément à prendre en compte dans la prise de décision est l'expression de préférences sur les éléments du formalisme, reflétant l'importance relative des buts, des règles ou des faits du système. Une façon de modéliser cet aspect dans le formalisme reste à étudier.

Comme précédemment indiqué, nous avons considéré un sous-ensemble du langage logique introduit par (Chein, Mugnier, 2009) afin de nous conformer au mieux au cas d'utilisation étudié. Il serait intéressant d'utiliser cette méthodologie dans d'autres scénarios où ce type de règles se présentent naturellement. Une première étape est bien sûr l'identification de tels scénarios. Puis les notions de dérivation inverse et dérivation avant doivent être étendues afin de permettre des dérivations potentiellement infinies (un résultat direct de l'introduction de variables existentielles en conclusion). La notion de minimalité telle qu'abordée par (Klarman et al., 2011) est alors essentielle.

Sur les aspects touchant l'argumentation, il serait également judicieux d'étudier les conséquences de l'expression de préférences sur les points de vue. Comment de telles préférences se traduiront en préférences entre arguments est encore une question ouverte.

\section{Bibliographie}

Amgoud L., Cayrol C. (2002). A reasoning model based on the production of acceptable arguments. Annals of Mathematics and Artificial Intelligence, vol. 34, p. 197-216.

Amgoud L., Parsons S., Perrussel L. (2000). An argumentation framework based on contextual preferences. In Proceedings of the international conference on formal and applied and practical reasoning, p. 59-67.

Amgoud L., Prade H. (2009). Using arguments for making and explaining decisions. Artificial Intelligence, vol. 173, no 3-4, p. 413-436. 
AQUANUP. (2009). http://www.inra.fr/inra_cepia/vous_recherchez/des_projets/france/ aquanup.

Baget J.-F., Salvat E. (2006). Rules dependencies in backward chaining of conceptual graphs rules. In Conceptual structures: Inspiration and application, 14th international conference on conceptual structures, vol. 4068, p. 102-116. Springer.

Bench-Capon T. J. M. (2003). Persuasion in practical argument using value-based argumentation frameworks. Journal of Logic and Computation, vol. 13, nº 3, p. 429-448.

Besnard P., Hunter A. (2008). Elements of argumentation. The MIT Press.

Bourguet J.-R. (2010). Contribution aux méthodes d'argumentation pour la prise de décision. application à l'arbitrage au sein de la filière céréalière. Thèse de doctorat, Université Montpellier II, Montpellier, France.

Bourguet J.-R., Thomopoulos R., Mugnier M.-L., Abécassis J. (2013). An artificial intelligencebased approach to deal with argumentation applied to food quality in a public health policy. Expert Systems With Applications, vol. 40, n 11, p. 4539-4546.

Bourre J.-M., Bégat A., Leroux M.-C., Mousques-Cami V., Pérandel N., Souply F. (2008). Valeur nutritionnelle (macro et micro-nutriments) de farines et pains français. Médecine et Nutrition, vol. 44, no 2, p. 49-76.

Bouyssou D., Dubois D., Pirlot M., Prade H. (2009). Decision-making process - concepts and methods. Wiley.

CADINNO. (2008). Information, choix, consommateurs responsables : des leviers pour un développement durable? http://www.melissa.ens-cachan.fr/IMG/pdf/Colloque_CadInno_FR .pdf.

Chein M., Mugnier M.-L. (2009). Graph-based knowledge representation and reasoning, computational foundations of conceptual graphs. Springer London.

Clocksin W. F., Mellish C. (1984). Programming in prolog, 2nd edition. Springer.

Dean M., Sheperd R., Arvola A., Lampila P., Lahteenmaki L. et al. (2007). Report on consumer expectations of health benefits of modified cereal products. Rapport technique. University of Surrey, UK.

DINABIO. (2008). Proceedings of Dinabio, Développement et innovation en agriculture biologique. http://www.inra.fr/ciag/revue_innovations_agronomiques/volume_4_janvier_2009.

Dubuisson-Quellier S. (2006). De la routine à la délibération. les arbitrages des consommateurs en situation d'achat. Réseaux, vol. 135/136, p. 253-284.

Dung P. M. (1995). On the acceptability of arguments and its fundamental role in nonmonotonic reasoning, logic programming and $n$-person games. Artificial Intelligence Journal, vol. 77 , p. 321-357.

FCN. (2009). Fibres, céréales et nutrition. http://www.inra.fr/content/view/full/24670029.

Ginon E., Lohérac Y., Martin C., Combris P., Issanchou S. (2009). Effect of fibre information on consumer willingness to pay for french baguettes. Food Quality and Preference, vol. 20, p. 343-352.

Gottsegen J. (1998). Using argumentation analysis to assess stakeholder interests in planning debates. Computers, Environment and Urban Systems, vol. 22, no 4, p. 365 - 379. 
HEALTHGRAIN. (2009). http://www.healthgrain.org.

Kaci S., Torre L. van der. (2008). Preference-based argumentation: Arguments supporting multiple values. International Journal of Approximate Reasoning, vol. 48, $\mathrm{n}^{\circ} 3, \mathrm{p} .730$ 751.

Klarman S., Endriss U., Schlobach S. (2011). Abox abduction in the description logic alc. J. Autom. Reasoning, vol. 46, n 1 , p. 43-80.

Layat T. (2011). Place du pain dans l'équilibre alimentaire. Pratiques en nutrition, vol. 7, $\mathrm{n}^{\circ} 26$, p. $45-50$.

Maguire L. A., Boiney L. G. (1994). Resolving environmental disputes: a framework incorporating decision analysis and dispute resolution techniques. Journal of Environmental Management, vol. 42, n 1, p. 31 - 48.

PNNS (documents statutaires). (2010). http://www.sante.gouv.fr/htm/pointsur/nutrition/pol _nutri4.htm.

PNNS (site web). (2010). http://www.mangerbouger.fr/menu-secondaire/pnns/le-pnns.

Rahwan I., Simari G. (2009). Argumentation in artificial intelligence. Springer.

Slavin J., Green H. (2007). Diatery fibre and satiety. British Nutrition Foundation, vol. 32(1), p. $32-42$.

Thomopoulos R., Charnomordic B., Cuq B., Abécassis J. (2009, September). Artificial intelligence-based decision support system to manage quality of durum wheat products. Quality Assurance and Safety of Crops \& Foods, vol. 1, n 3, p. 179-190.

Van Melle W., Scott A., Bennett J., Peairs M. (1981). The EMYCIN manual. Stanford University Press.

Vesic S. (2012). Maxi-consistent operators in argumentation. In L. D. Raedt et al. (Eds.), Ecai, vol. 242, p. 810-815. IOS Press. 
